\title{
Molecular characterization of swine leucocyte antigen class I genes in outbred pig populations
}

\author{
C.-S. Ho ${ }^{*,+}$, , J. K. Lunney ${ }^{\S}$, M. H. Franzo-Romain*, G. W. Martens ${ }^{\dagger, \neq, 1}$, Y.-J. Lee ${ }^{\Uparrow}$, J.-H. Lee ${ }^{\natural}$, \\ M. Wysocki ${ }^{\S}$, R. R. R. Rowland** and D. M. Smith*, ${ }^{*}$, \\ * Department of Pathology, University of Michigan, Ann Arbor, MI 48109, USA. 'Transplant Immunology Laboratory, Department of \\ Pathology, Baylor University Medical Center, Dallas, TX 75246, USA. ${ }^{\ddagger}$ Institute of Biomedical Studies, Baylor University, Waco, TX 76798 , \\ USA. ${ }^{\S}$ APDL, BARC, ARS, USDA, Beltsville, MD 20705, USA. "Division of Animal Science and Resources, College of Agriculture and Life \\ Sciences, Chungnam National University, Daejeon 305-764, Korea. * ${ }^{*}$ Department of Diagnostic Medicine and Pathobiology, College of \\ Veterinary Medicine, Kansas State University, Manhattan, KS 66506, USA
}

\section{Summary}

\begin{abstract}
The highly polymorphic swine leucocyte antigen (SLA) genes are one of the most important determinants in swine immune responses to infectious diseases, vaccines, and in transplantation success. Study of SLA influence requires accurate and effective typing methods. We developed a simple and rapid method to type alleles at the three classical SLA class I loci (SLA-1, SLA-3 and SLA-2) using the PCR-sequence-specific primer (PCR-SSP) strategy. This typing system relies on 47 discriminatory PCR primer pairs designed to amplify the SLA class I alleles by groups that have similar sequence motifs. We applied this low-resolution group-specific typing method to characterize the SLA class I alleles present in three outbred pig populations $(n=202)$. Alleles from 24 class I allele groups corresponding to 56 class I genotypes were detected. We also identified 23 low-resolution SLA class I haplotypes in these pigs and found haplotypes Lr-1.0 (SLA-1*01XX-SLA-3*01XX-SLA-2*01XX) and Lr-4.0 (SLA-1*04XX-SLA-3*04XX-SLA-2*04XX) in all three pig populations with a high prevalence. Over $80 \%$ of the pigs examined $(n=162)$ were found to bear at least one of these haplotypes, resulting in a combined haplotype frequency of nearly 50\%. This PCRSSP-based typing system demonstrates a reliable and unambiguous detection of SLA class I alleles, and can be used to effectively investigate the SLA diversity in outbred pig populations. It will help to identify the role of SLA antigens in disease-resistant pigs and may facilitate the development of effective vaccines.
\end{abstract}

Keywords genotyping, major histocompatibility complex, outbred pigs, PCR-SSP, polymorphism, SLA, SLA diversity, swine leucocyte antigen.

\section{Introduction}

The porcine major histocompatibility complex (MHC), which codes for the swine leucocyte antigens (SLA), has been associated with many immunological, pathological and physiological conditions. The SLA genes have been

Address for correspondence

D. M. Smith, Department of Pathology, University of Michigan, Ann Arbor, MI 48109, USA.

E-mail: dousmith@umich.edu

${ }^{1}$ Present address: Department of Medicine, University of Massachusetts School of Medicine, Worcester, MA 01655, USA.

Accepted for publication 28 December 2008 repeatedly shown to influence swine immune traits (Mallard et al. 1989a,b), disease resistance (Lacey et al. 1989; Madden et al. 1990), vaccine responsiveness (Rothschild et al. 1984; Lumsden et al. 1993) and tumour penetrance (Tissot et al. 1989) (reviewed in Vaiman et al. 1998 and Lunney et al. 2008). A number of favourable production traits have also been associated with the SLA region (reviewed in Vaiman et al. 1998); recent finer mapping may separate these quantitative trait loci (QTL) from the SLA loci (Demeure et al. 2005). The strong influence of the SLA complex is mostly attributable to the antigen-presenting properties of MHC proteins in the adaptive immune system, as well as many important genes that are in strong linkage (Renard et al. 2006; 
Lunney et al. 2008). Therefore, understanding the SLA genes is crucial to the improvement of animal health in the pig production industry and the development of pigs as large animal models for human diseases.

As with most mammalian MHC systems, the SLA system is highly polymorphic. At least 72 serologically defined SLA class I haplotypes (designated H01-H72) have been reported (Renard et al. 1988; Chardon et al. 2000). However, this number may be an underestimate due to the detection limits of older serologic reagents. The majority of these haplotypes were characterized in the commercial pig breeds in Europe and may not be representative of the global pig population. According to the Immuno Polymorphism Database (IPD)-MHC SLA sequence database (http://www.ebi.ac.uk/ipd/mhc/sla/), there are 116 classical SLA class I (SLA-1, SLA-3 and $S L A-2)$ alleles, and 28 haplotypes of these loci have been designated to date by the SLA Nomenclature Committee of the International Society for Animal Genetics (ISAG) (Robinson et al. 2005; Smith et al. 2005b; Ellis et al. 2006; Ho et al. 2009).

Due to the extensive polymorphic nature of SLA genes, accurate and sensitive typing methods are crucial for studying their influences. Serologic typing using alloantisera has historically been the most important means for determining SLA class I antigen specificities (Renard et al. 1988). However, the lack of typing sera with well-defined specificities often creates problems, as many animals frequently have untyped or 'blank' SLA antigens. MHC molecules often share similar epitopes that can be bound by the same antibody, making most SLA typing sera highly cross-reactive. Thus, a combination of typing sera is often required to differentiate between animals. Most typing reagents are also directed against an entire haplotype rather than individual alleles, making their resolution undesirable. Furthermore, serologic typing has inherent limitations in its ability to distinguish between alleles that differ at sites inaccessible to antibody binding (e.g. epitopes that are buried within the SLA proteins).

The aim of this study was to develop a simple and costeffective typing system that can be used to examine the SLA class I allelic diversity in outbred pig populations. Here, we describe a low-resolution DNA-based typing method using the PCR-sequence-specific primer (PCR-SSP) strategy to type alleles at three classical SLA class I loci. We have shown that this method of typing allows an accurate characterization of the SLA class I alleles and the prevalent class I haplotypes present in three populations of outbred commercial pigs. We suggest that it can be used effectively to investigate the SLA diversity in other outbred pig populations and may facilitate the development of more effective vaccines and the identification of disease-resistant pigs in the context of SLA antigens.

\section{Materials and methods}

\section{Sequence-specific primers and typing setup}

The majority of the classical SLA class I alleles (SLA-1, SLA-3 and $S L A-2)$ that have been defined by DNA sequencing were previously assigned into allele groups based on sequence similarity and phylogenetic analysis by the ISAG SLA Nomenclature Committee (Smith et al. 2005b). Several tentative alleles remain unassigned due to distinctive sequence motifs (e.g. SLA-2*es22) and/or insufficient sequence data (incomplete exons 2 and 3) (e.g. SLA-1*an01). To differentiate alleles by groups that share similar sequence motifs (i.e. low-resolution/group-specific typing), a set of 47 sequencespecific PCR primer pairs was developed based on polymorphisms in exons 1, 2 and 3 of the published sequences using the alignment tool available at the IPD-MHC SLA website (Table 1). This also included primer pairs designed to detect independently the tentative alleles without group designation. All typing primers were validated using stored DNA samples from pigs and cell lines with previously defined SLA specificities (Lee et al. 2005; Smith et al. 2005c; Ho et al. 2006; C.-S. Ho et al., unpublished data).

PCR reactions were set up in $10-\mu \mathrm{l}$ reaction volumes as previously described, with the internal positive control primers specific for the porcine $\alpha$-actin gene (ACTA1) and $\sim 30 \mathrm{ng}$ of DNA in each reaction (Martens et al. 2003; Ho et al. 2006). A 5- $\mu$ l reaction volume has recently been adopted to further reduce the amount of DNA required and the cost of the reagents. All primers were optimized to be used at the same concentration (5 pmol/each) and annealing temperature $\left(65^{\circ} \mathrm{C}\right)$. Typing of each pig included a negative control without DNA to check for reagent contamination, and was set up and electrophoresed in a standard 96-well format. Interpretation of the results was based on the presence of a smaller PCR product than the positive control in each lane.

\section{Designation of low-resolution SLA allele specificity}

In parallel with the naming conventions for the DNAsequenced SLA alleles (Smith et al. 2005a,b), the SLA class I allele specificities characterized by the low-resolution groupspecific SLA typing were defined with the first two digits to indicate groups of alleles with similar sequence motifs and the third and fourth digits as ' $\mathrm{XX}$ ' to indicate low-resolution group specificity (e.g. $S L A-1^{*} 01 \mathrm{XX}$ ). Names of the tentative alleles without group designation were used directly to represent their specificity (e.g. SLA-1*rhO3).

\section{Designation of low-resolution SLA haplotype}

Given the strong linkage between SLA loci, it is often more appropriate and convenient to present genotype data in the form of haplotypes. The low-resolution SLA allele 
Ho et al.

Table 1 Sequence-specific primers for typing SLA class I alleles

\begin{tabular}{|c|c|c|c|c|c|}
\hline \multirow[b]{2}{*}{ Lane } & \multicolumn{2}{|l|}{ Specificity } & \multirow{2}{*}{$\begin{array}{l}\text { Primer sequence }\left(5^{\prime} \rightarrow 3^{\prime}\right) \\
(\mathrm{F}: \text { forward; R: reverse) }\end{array}$} & \multirow{2}{*}{$\begin{array}{l}\text { Primer } \\
\text { position }^{3}\end{array}$} & \multirow{2}{*}{$\begin{array}{l}\text { Product } \\
\text { size (bp) }\end{array}$} \\
\hline & Group $^{1}$ & Allele ${ }^{2}$ & & & \\
\hline \multirow[t]{2}{*}{1} & Negative control & & F: CGCCATGTGTGACGAAGACGAGACC & +21 & 516 \\
\hline & (ACTA1) & & R: CACGTACATGGCGGGCACGTTGAAG & +384 & \\
\hline \multirow[t]{2}{*}{2} & $S L A-3 * 01 X X$ & All: 0101/01ev04/01rh12/01rh28 & F: CCTCCGCGGGTACAGTCAGTTT & +411 & 174 \\
\hline & & & R: CACTTCACACACGCGCCCTC & +544 & \\
\hline \multirow[t]{2}{*}{3} & $S L A-3 * 03 X X$ & $0301 \sim 0304 / 03 a n 02 / 03 a n 04 / 03 a n 05^{4}$ & F: GCTGGAAGCCCCGTTTCATCT & +133 & 209 \\
\hline & & & R: GTTGTAGTAGCCGCGCAGGTTC & +300 & \\
\hline \multirow[t]{2}{*}{4} & $S L A-3{ }^{*} 04 X X$ & All: $0401 \sim 02 / 04 \mathrm{es} 32$ & F: GGAAGCCCCGTTTCATCGAA & +135 & 192 \\
\hline & & & R: GCAGGTTTTTCAGGTTCACTCGGA & +284 & \\
\hline \multirow[t]{2}{*}{5} & $S L A-3 * 05 X X$ & All: 0501 03/05sw01 & F: CGTGGAAGATACGCAGTTCGTGT & +166 & 139 \\
\hline & & & R: AGTCTGTGCGTTGTCCTTGCTGA & +260 & \\
\hline \multirow[t]{2}{*}{6} & $S L A-3 * 06 X X$ & 0601 & F: GCGACGTCGGGCCAGACT & +382 & 153 \\
\hline & & & R: CATCGGCCGCCTCCCA & +502 & \\
\hline \multirow[t]{2}{*}{7} & $S L A-3 * 06 X X$ & 0602 & F: GCAGGAAGCCCCGTTTCAC & +131 & 140 \\
\hline & & & R: CTCСTCATCССААТАСТССТGССT & +229 & \\
\hline \multirow[t]{2}{*}{8} & $S L A-3 * 07 X X$ & All: 0701/0701sm19/07Lw02/07rh34 & F: CGACCGCAGGAAGCCCCGT & +126 & 153 \\
\hline & & & R: TCCTCATCCCAATACTCCTGCCA & $+238^{5}$ & \\
\hline \multirow[t]{2}{*}{9} & $S L A-3^{*}$ & $\mathrm{hm} 22$ & F: GGGTCTCACACCATCCAGGT & +353 & 210 \\
\hline & & & R: CAGGTAGCTCCTCCTACGCTCTGT & +520 & \\
\hline \multirow[t]{2}{*}{10} & $S L A-3 * 01 X X$ & 0101/01ev04/01rh28 & F: TCGCGGGTACAGTCAGTTTGG & +413 & 193 \\
\hline & & & R: CATCTTCAGGTATTTCTGGAGCCACA & +560 & \\
\hline \multirow[t]{2}{*}{11} & $S L A-3 * 03 X X$ & 0301/0304/03an04/03an05 & F: GCTGGTAGCCCCGTTTCATCT & +133 & 149 \\
\hline & & & R: GTTCTTCGTCTCCTCATCCCAATACTT & +235 & \\
\hline \multirow[t]{3}{*}{12} & $S L A-3 * 03 X X$ & 0301 04/03an02/03an04/03an05 & F: TCCCCACTCCCTGAGGTATTTCG & +88 & 139 \\
\hline & $S L A-3 * 04 X X$ & All: $0401 \sim 02 / 04 \mathrm{es} 32$ & R: CGGCTCCATCTGCGGATTG & +186 & \\
\hline & $S L A-1 * 11 X X$ & 11jh02 & & & \\
\hline \multirow[t]{2}{*}{13} & $S L A-3 * 04 X X$ & 0402/04es32 & F: CGACCGCAGGAAGCCCCGT & +126 & 215 \\
\hline & & & R: GGTTGTAGTAGCCGCGCAGC & +303 & \\
\hline 14 & $S L A-3 * 05 X X$ & $0502 \sim 03 / 05 s w 01$ & F: GTGGACGACTCGCAGTTCGTGT & +166 & 114 \\
\hline & & & R: GTCTCCCGATCCCAATACTCCTGA & +231 & \\
\hline 15 & $S L A-3 * 06 X X$ & All: $0601 \sim 02$ & F: GCGACGTCGGGCCAGACT & +382 & 188 \\
\hline & $S L A-3 * 07 X X$ & 0701sm19/07rh34 & R: CGCGCCCTCCAGGTAGCTT & +534 & \\
\hline 16 & $S L A-3 * 06 X X$ & 0602 & F: CGACGTGGGRCCAGACT & +382 & 172 \\
\hline & $S L A-3 * 07 X X$ & 0701/0701sm19/07Lw02 & R: GCTTCTCATTTGCTCCGCCG & +518 & \\
\hline 17 & $S L A-2 * 01 X X$ & All: 0101/01an06 & F: TCCССАСТСССTGAGCTATTTCTC & $+89^{6}$ & 256 \\
\hline & $S L A-1^{*}$ & es11 & R: GTTGTAGTAGCCGCGCAGGGTC & $+300^{6}$ & \\
\hline 18 & $S L A-2 * 02 X X$ & All: $0201 \sim 02$ & F: CCTCCGCGGGTACAGTCAGTTT & +420 & 139 \\
\hline & & & R: CTGCTCCGCCACATTGGCT & +519 & \\
\hline 19 & $S L A-2 * 03 X X$ & All: 0301 02/03gz01 & F: GCAGTTCGTGCGGTTCGACAGC & $+177^{6}$ & 134 \\
\hline & $S L A-1^{*}$ & an01/an02 & R: CTCGGTAAGTCTGTGAGGTGTCCCTTTGTA & $+260^{6}$ & \\
\hline & $S L A-2^{*}$ & es22 & & & \\
\hline 20 & $S L A-2 * 04 X X$ & All: 0401/040201 02 & F: CCGAGGGAACCTGCGCACAGC & +314 & 316 \\
\hline & & & R: CCCACGTCGCAGCCGTACATGA & +362 & \\
\hline 21 & $S L A-2 * 05 X X$ & All: 0501 02/05rh03/05rh07/ & F: CGGGCGCCGTGGATAGAGA & +232 & 127 \\
\hline & & 05rh34/05sy01 & R: CCTCGCTCTGGTTGTAGTAGCCAAG & +316 & \\
\hline 22 & $S L A-2 * 06 X X$ & All: 0601/06an03/06me01/ & F: CGCCCCGAATCCGAGGAAA & +207 & 127 \\
\hline & & 06sr01/06sv01 & R: CAGGGTGTTCAGGTTCACTCGGTA & +292 & \\
\hline 23 & $S L A-2 * 07 X X$ & All: 0701/07an05/07rh12/07we01 & F: GTCATGGTCTCACACCCTCCAGGT & +362 & 199 \\
\hline & & & R: TCCCTCCGCCACATTGGCT & +519 & \\
\hline 24 & $S L A-2 * w 08 X X$ & All: w08gx01/w08sw01 & F: CGCCCCGAATCCGAGGAAA & +207 & 126 \\
\hline & & & R: AGGGTCTTCAGGTCCACTCGGTT & +292 & \\
\hline 25 & $S L A-2 *$ w09XX & All: w09an02/w09pt22/w09sn01 & F: TGTGGGACCAGACGGGCTCT & +397 & 181 \\
\hline & & & R: GCCTTGCAGGTAGCTCCTCCAG & +537 & \\
\hline 26 & $S L A-2 * 10 X X$ & All: 1001/10an01/10es21/10sk21/ & F: GCCTCGACACAGAATCTCCGCA & -11 & 116 \\
\hline & & 10sm01 & R: CCCGCACTCACCCGCCTGA & +66 & \\
\hline
\end{tabular}


Table 1 Continued.

\begin{tabular}{|c|c|c|c|c|c|}
\hline \multirow[b]{2}{*}{ Lane } & \multicolumn{2}{|l|}{ Specificity } & \multirow{2}{*}{$\begin{array}{l}\text { Primer sequence }\left(5^{\prime} \rightarrow 3^{\prime}\right) \\
(\mathrm{F}: \text { forward; R: reverse) }\end{array}$} & \multirow{2}{*}{$\begin{array}{l}\text { Primer } \\
\text { position }^{3}\end{array}$} & \multirow{2}{*}{$\begin{array}{l}\text { Product } \\
\text { size }(b p)\end{array}$} \\
\hline & Group $^{1}$ & Allele ${ }^{2}$ & & & \\
\hline \multirow[t]{2}{*}{27} & $S L A-2 * 11 X X$ & All: 110101 02/11so01 & F: GACGCTCCGAATCCGAGGGA & +206 & 126 \\
\hline & & & R: TGTGCGCAGGTACCCTCTGTAAA & +290 & \\
\hline \multirow[t]{2}{*}{28} & $S L A-2 * 12 X X$ & All: 1201/12Lw01 & F: CCTCCGCGGGTACAGTCAGTTC & $+411^{6}$ & 160 \\
\hline & $S L A-1^{*}$ & es11 & R: GCCTTGCAGGTAGCTCCTCCAG & $+528^{6}$ & \\
\hline \multirow[t]{2}{*}{29} & $S L A-2 * w 13 X X$ & All: $w 13 s m 20$ & F: TMGARMAGGAGGGGCAGGG & $+236^{6}$ & 117 \\
\hline & $S L A-1 * 11 X X$ & 11jh02 & R: CGGGCTCGCTCTGGTTGTAGTA & $+313^{6}$ & \\
\hline \multirow[t]{2}{*}{30} & $S L A-2 * w 14 X X$ & All: w14yn01 & F: TCCTCCGCGGGTACAGTCAGTA & +419 & 161 \\
\hline & & & R: GCCCTGCAGGTAGCTCCTCATT & +537 & \\
\hline \multirow[t]{2}{*}{31} & $S L A-2^{*}$ & an04 & F: GACCTCTGTGACTCCCGCTTCC & +139 & 143 \\
\hline & & & R: TTCCTATCCCAATACTCCTGCCCT & +237 & \\
\hline \multirow[t]{2}{*}{32} & $S L A-2^{*}$ & jh01 & F: CGACCKCAGGAAGCCCCGT & $+126^{6}$ & 203 \\
\hline & $S L A-3^{*}$ & $\mathrm{hm} 22$ & R: CGCGCAGGKTGTTCAGGC & $+293^{6}$ & \\
\hline \multirow[t]{2}{*}{33} & $S L A-1 * 01 X X$ & All: $0101 \sim 02 / 01 \mathrm{rh} 28$ & F: CGGGGTCAGGGTCTCACACCTA & +347 & 209 \\
\hline & & & R: CСТCCTACGCTCCGCCACATT & +514 & \\
\hline \multirow[t]{2}{*}{34} & $S L A-1 * 02 X X$ & All: 0201 02/02we02 & F: GCAGTTCGTGCGGTTCGACAACT & +178 & 148 \\
\hline & & & R: GGTGTTCAGGCCCACTCGGAG & +283 & \\
\hline \multirow[t]{2}{*}{35} & $S L A-1 * 04 X X$ & All: 0401/04gx01/04gz01/04we01 & F: GCCTGACCGCGGGGACTCT & +123 & 181 \\
\hline & & & R: GTAAGTCTGTGCGGTTTCCTTGACA & +261 & \\
\hline \multirow[t]{2}{*}{36} & $S L A-1 * 05 X X$ & All: 0501 & F: ССССАСТСССТСАGСТАТTTСТC & +89 & 223 \\
\hline & $S L A-1^{*}$ & an02 & R: CTCGGAAAGTCTGTGAGGTGTCCC & +266 & \\
\hline \multirow[t]{2}{*}{37} & $S L A-1 * 06 X X$ & All: 0601/06an04 & F: GCCTGACCGCGGGGACTCT & +123 & 164 \\
\hline & $S L A-1 * 13 X X$ & 1301 & R: CCTTGACTTTCCGCGTCTCCTC & +247 & \\
\hline \multirow[t]{2}{*}{38} & $S L A-1 * 07 X X$ & All: $0701 \sim 02$ & F: GCCGGGTCTCACACCATCCAGAT & +353 & 220 \\
\hline & & & R: GGCCCTGCAGGTAGCTCCTCAAT & +528 & \\
\hline \multirow[t]{2}{*}{39} & $S L A-1 * 08 X X$ & $\begin{array}{l}\text { All: 0801/08an031/08Lw02/ } \\
\text { 08ms05/08pt13/ }\end{array}$ & F: CGTGGACTCCCGCTTCTTCATT & +135 & 141 \\
\hline & & 08sk11/08sm08/08sy01 & R: GTCTCCCGATCCCAATACTCCG & +233 & \\
\hline \multirow[t]{2}{*}{40} & $S L A-1{ }^{*}$ w09XX & All: w09sm09 & F: ССАСТСССТGАGСТАТTTСTT & +89 & 193 \\
\hline & & & R: GATCTGTGTCTCCCGATCCCAATAG & +237 & \\
\hline \multirow[t]{2}{*}{41} & $S L A-1{ }^{*} \mathrm{w} 10 \mathrm{XX}$ & All: w10cs011/w10sm21 & F: СTCСтCСTCCGCGGGTACGA & +404 & 180 \\
\hline & & & R: CCACTCCACACACGTGCCCTC & +544 & \\
\hline \multirow[t]{2}{*}{42} & $S L A-1 * 11 X X$ & All: 1101/11jh01/11jh02/11mp11/11yn01 & F: GTGTCCCGGCCCGACC & +112 & 183 \\
\hline & & & R: CTGTGCGCTGCCCATGACAC & +260 & \\
\hline \multirow[t]{2}{*}{43} & $S L A-1 * 12 X X$ & All: 1201/12hy01/12Lw01 & F: GGTTCGACAGCGACGCCCTC & +186 & 121 \\
\hline & & & R: CGGTTAATCTGTGCGGTTTCCTTGA & +263 & \\
\hline \multirow[t]{2}{*}{44} & $S L A-1 * 13 X X$ & All: $1301 / 13 m s 21$ & F: GTCTCACACCCTCCAGAGCATGTTT & +360 & 217 \\
\hline & & & R: CAGTCCCTGCAGGTAGCTCCTCCTA & +528 & \\
\hline 45 & $S L A-1^{*}$ & rh03 & F: GGTACAGWCAGTAYGSCTACGACA & $+421^{6}$ & 193 \\
\hline & $S L A-2^{*}$ & es22 & R: TTCCCCATCTCCAGGTATCTGC & $+569^{6}$ & \\
\hline 46 & $S L A-1^{*}$ & an01 & F: CGTGGACTCCCGCTTCTTCATT & +135 & 173 \\
\hline & & & R: GGAAAGTCTGTGAGGTGTCCCTTTG & +262 & \\
\hline 47 & $S L A-1^{*}$ & an02 & F: ССССАСТСССТСАGСТАТТTСТС & +89 & 172 \\
\hline & & & R: ACTCCTGCСССTCCTGTTCG & +219 & \\
\hline 48 & $S L A-1^{*}$ & $\operatorname{cs} 02$ & F: CTCACACCCTCCAGAGCATGTTT & +360 & 209 \\
\hline & & & R: CTGCAGGTAGCTCСTCTCTCC & +526 & \\
\hline
\end{tabular}

SLA, swine leucocyte antigen.

${ }^{1}$ Specificity without group designation indicates tentative allele that has not been assigned to group due to distinctive sequence motifs and/or insufficient sequence data.

2،All' indicates all the published allele(s) that have been assigned to the group (Smith et al. 2005b; Ho et al. 2009).

${ }^{3}$ Refers to the nucleotide position of SLA genes corresponding to the 3 ' terminus of the primer.

${ }^{4}$ Does not include new allele $S L A-3{ }^{*} 03 p t 31$.

${ }^{5}$ Located in the 9-bp insertion unique to $S L A-3 * 07 X X$ allele group.

${ }^{6}$ Add 9 bp for the nucleotide position of the SLA-2 specificities. 
specificities characterized in this study were assigned into haplotypes based on their inheritance and segregation in descendents when pedigree was available. Otherwise, the most probable SLA haplotypes were deduced using a series of comparative and subtractive approaches based on the presence of SLA homozygous animals within the sample population, the frequency of occurrence of individual alleles as haplotypes within the population, data comparisons to the published high-resolution SLA class I haplotypes (Smith et al. 2005b; Ho et al. 2009), and the class I haplotypes recently identified in nearly 650 pedigreed pigs obtained from multiple commercial sources using the typing method as described here (C.-S. Ho, unpublished data). The SLA class II genotypes of these pigs (as determined by a typing method using a similar approach) are not reported here but were also taken into consideration for resolving ambiguities.

In parallel to the naming conventions for high-resolution DNA-sequenced SLA haplotypes (Hp-) (Smith et al. 2005a,b), the low-resolution SLA haplotypes identified in this study were tentatively designated with a prefix 'Lr-' and a number for the class I haplotype followed by a number for the class II haplotype separated by a period (e.g. Lr-1.1). The class II haplotypes are not reported here and have all been designated with the number ' 0 '. The low-resolution haplotypes were numbered according to the high-resolution haplotypes if the allele group assignments were identical (e.g. Lr-1.0, SLA-1*01XX-SLA-3*01XX-SLA-2*01XX vs. Hp-1.0, SLA-1*0101-SLA-3*0101-SLA-2*0101). New haplotypes were arbitrarily designated with new numbers if similarities were not observed.

\section{Experimental pig populations}

Three experimental populations of outbred pigs with unknown immunogenetic background totalling 202 animals were evaluated in this study for their SLA class I allele diversity. The first pig population (arbitrarily designated the KSU pigs) consisted of 49 animals derived from five pedigreed families which included the nine founding animals (four boars and five sows) and their offspring $(n=40)$. The founding animals were crossbred commercial pigs (Pig Improvement Company, PIC) but their relatedness was not known. This herd was established for a study on swine genetic resistance to the porcine respiratory and reproductive syndrome virus (PRRSV) (C.R. Wyatt, unpublished data).

The second pig population [arbitrarily designated the porcine circovirus (PCV) pigs] consisted of 52 non-pedigreed germ-free pigs established for a study on swine genetic susceptibility to subgroups of PCV type 2 (Lager et al. 2007). Their relatedness and origin were not known.

The third pig population (designated Big Pig) consisted of 101 non-pedigreed piglets derived from 17 litters of commercial Large White/Landrace crosses. The relatedness between these pigs was also unknown. These pigs were included in another multi-institutional, multi-disciplinary study on persistence of PRRSV infection (R.M. Molina et al., unpublished data).

\section{Results}

\section{Low-resolution SLA-typing primers}

Forty-seven sequence-specific PCR primer pairs (which amplified products of 114-316 bp) were designed based on the published SLA sequences to type pigs at SLA-1, SLA-3 and SLA-2 (Table 1). These typing primers were developed to differentiate unambiguously between alleles by groups that share polymorphic sequence motifs. Allele-specific primers were also designed to detect independently the tentative alleles without group designation and the alleles from groups in which no group-specific sequence motif could be identified (i.e. lane 6, SLA-3*0601; lane 7, SLA3*0602). In addition, seven 'medium-resolution' primer pairs (lane 10-16) were added to differentiate subgroups of SLA-3 alleles (e.g. the primer pair in lane 11 will not amplify SLA-3*0302/0303/03an02/03pt31). This arrangement (47 primer pairs and a negative control) allows the typing of two animals to be efficiently set up in a standard 96-well PCR tray. The specificities of the typing primers used in this study covered the majority of the SLA class I alleles published to date $(111 / 116)$. Five new alleles (SLA-1*es12, SLA-1*st11, SLA-1*sk13, SLA-2*jh02 and SLA-3*03pt31) were not included due to their recent addition to the database as well as their distinctive sequence motifs that were not assayed.

\section{Primer sensitivity and specificity}

DNA samples from five SLA-defined resource pig populations representing six breeds were used to validate the sensitivity and specificity of the typing primers. These included samples from the National Institutes of Health (NIH) and the Yucatan miniature pigs (Smith et al. 2005c), the Sinclair $\times$ Hanford crossbred miniature pigs (C.-S. Ho et al., unpublished data), the Westran pigs (Lee et al. 2005) and the Meishan pigs (Ho et al. 2006). Eight commercially available porcine cell lines (American Type Culture Collection, ATCC) that had been SLA-typed by the sequence-based typing (SBT) method (C.-S. Ho et al., unpublished data) were also used for primer validation. Altogether, these reference samples represented 30 of the 32 SLA class I allele groups (12/12 SLA-1 groups, 6/6 SLA-3 groups and 12/14 SLA-2 groups) and seven of the 13 tentative alleles recognized to date. The reference samples also represented at least 18 of the 29 class I haplotypes that have been molecularly defined, of which six have also been suggested to harbour duplicated SLA-1 loci (Hp-2.0, -8.0, -11.0, -12.0, -19.0 and -20.0). The only representative control samples that were not available for validating the primers were those in lane 
30 (SLA-2*w14XX), lane 31 (SLA-2*an04), lane 46 (SLA-1*an01) and lane 47 (SLA-1*an02). Repeated evaluations indicated that the typing primers used in this study are sensitive and specific. The genotypes in the reference DNA samples could all be assigned accurately and unambiguously (data not shown). Weak non-specific bands were rarely observed and did not interfere with result interpretation. An example of the low-resolution SLA class I PCR-SSP typing is shown in Fig. 1.

\section{SLA class I diversity in the experimental outbred pigs}

SLA class I diversity was evaluated in the three experimental outbred pig populations totalling 202 animals. Fourteen class I genotypes were observed in the KSU pigs, 22 in the PCV pigs and 33 in the Big Pig samples, making a total of 56 unique class I genotypes (Table 2). These included alleles from 24 of the 32 SLA class I allele groups (8/12 SLA-1 allele groups, 6/6 SLA-3 allele groups and 10/14 SLA-2 allele groups) and a tentative allele without group designation (SLA-1*rh03).

Inheritance and segregation of the alleles in descendents revealed the presence of seven SLA class I haplotypes in the nine founding KSU pigs (Table 3; pedigree not shown). On the other hand, 13 and 16 class I haplotypes were deduced in the PCV pigs and the Big Pig samples, respectively, based on the frequency of occurrence of alleles as haplotypes within the population (Table 3). Overall this makes a total of 23 unique SLA class I haplotypes observed in this study. Twelve haplotypes (Lr-1.0, -2.0, -4.0 to -7.0, -11.0, -21.0, $-25.0,-27.0,-28.0$ and -59.0$)$ appeared to have highresolution counterparts as designated by the SLA
Nomenclature Committee and were therefore numbered accordingly.

Results indicated that haplotypes Lr-1.0, -4.0, -22.0, -25.0 and -27.0 were present in all three pig populations; Lr-21.0, -28.0 and -31.0 were present in the PCV pigs and the Big Pig samples. Lr-1.0 and Lr-4.0 were the two most common haplotypes among all three pig populations; their combined frequencies were found to be $58 \%$, 39\% and $47 \%$ in the KSU pigs, the PCV pigs and the Big Pig samples respectively. Altogether, 162 of the 202 pigs had one or both of the Lr-1.0 and Lr-4.0 haplotypes, accounting for $48 \%$ of the total number of SLA class I haplotypes detected in these pigs.

The typing primers used in this study were not able to detect the SLA-1 allele of Lr-29.0, -31.0, -34.0, -39.0 and -61.0, or the SLA-2 allele of Lr-59.0. Further testing with allele-specific primers revealed the presence of SLA-2*jhO2 (an allele that was not assayed) in Lr-59.0 (results not shown), and this haplotype was therefore numbered according to its high-resolution equivalent Hp-59.0 (Lee et al. 2008). No further attempts have been made to characterize the undetected SLA-1 specificities. In addition, the SLA-1 specificity of Lr-33.0 could not be determined due to ambiguity with the allele from the other haplotype (i.e. SLA-1*01XX of Lr-1.0) as it was only identified in one Big Pig sample (genotype 3; Table 2). We also noticed that Lr-2.0 was consistently amplified with two primer pairs specific for the SLA-3*04XX alleles (lanes 4 and 12; Table 1). The same reaction patterns were also observed with its high-resolution counterpart $\mathrm{Hp}-2.0$ in the $\mathrm{NIH}$ minipigs after repeated testing (data not shown). As previous studies showed that the SLA-3 gene was not expressed

Figure 1 Low-resolution SLA class I typing by the PCR-SSP method. Sequence-specific primers (which amplified products of 114-31$6 \mathrm{bp}$ ) were designed to differentiate SLA class I alleles by groups that have similar sequence motifs based on the published sequences (Smith et al. 2005b; Ho et al. 2009) (Table 1). Positive internal control primers (porcine $\alpha$-actin gene ACTA1; $516 \mathrm{bp}$ ) are multiplexed into each reaction to check for adequate amplification. The presence of a smaller (lower) band than the positive control band indicates that the reaction is positive. A negative control is set up without DNA to check for reagent contamination (lane 1). The pig sample shown here is heterozygous for SLA class I haplotype Lr-4.0/25.0 (Table 3), with specific amplifications in lane 3 and 12 (SLA-3*03XX), lane 4 and $12\left(S L A-3^{*} 04 X X\right)$, lane $20\left(S L A-2^{*} 04 X X\right)$, lane $23(S L A-2 * 07 X X)$, lane $35(S L A-1 * 04 X X)$ and lane $42(S L A-1 * 11 X X)$.

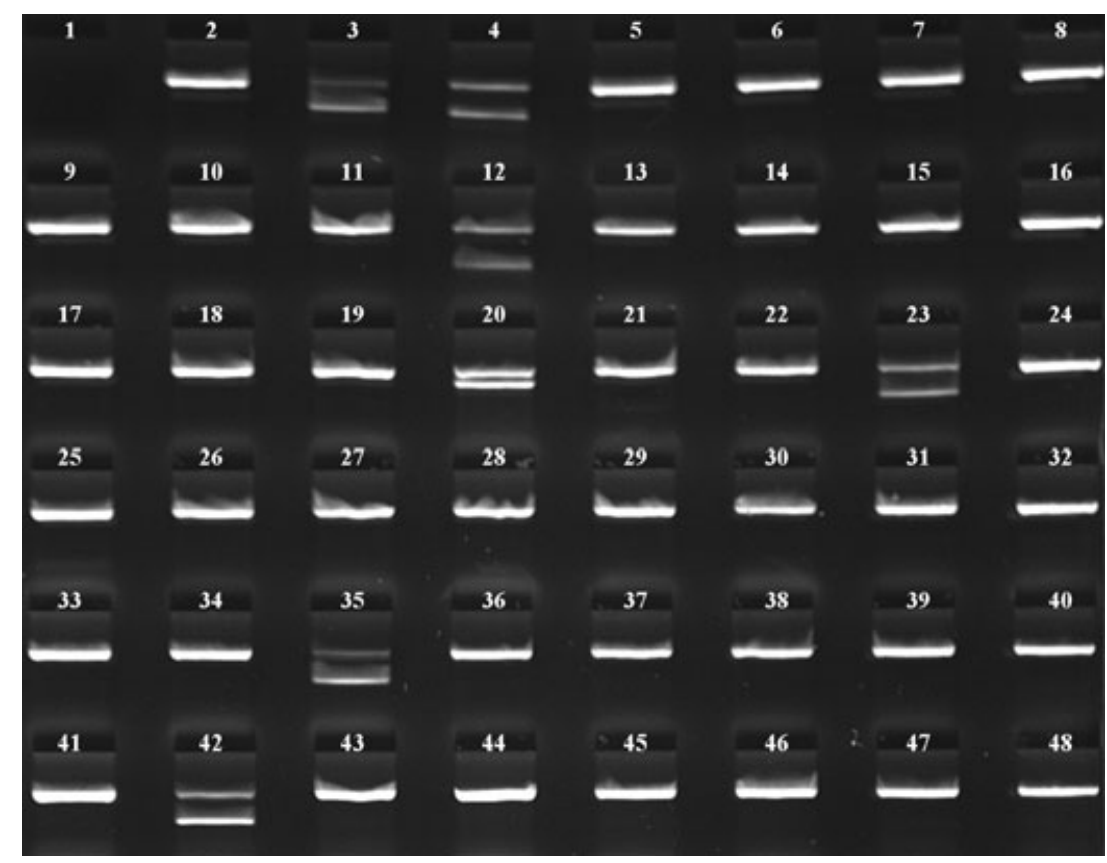


Ho et al.

Table 2 SLA class I genotypes characterized in three experimental populations of outbred pigs.

\begin{tabular}{|c|c|c|c|c|c|c|c|c|}
\hline \multirow{2}{*}{$\begin{array}{l}\text { SLA class I } \\
\text { genotype }\end{array}$} & \multicolumn{3}{|l|}{ Allele specificity ${ }^{1}$} & \multicolumn{4}{|c|}{ No. of pigs } & \multirow{2}{*}{$\begin{array}{l}\text { Corresponding SLA } \\
\text { class I haplotype }\end{array}$} \\
\hline & $S L A-1$ & $S L A-3$ & $S L A-2$ & KSU & PCV & Big Pig & Total & \\
\hline 1 & $01 X X$ & $01 \times x$ & $01 X X$ & 1 & & 7 & 8 & Lr-1.0/1.0 \\
\hline 2 & $01 X x$ & $01 X X, 03 X X$ & $01 X X, 06 X X$ & & 1 & & 1 & Lr-1.0/61.0 \\
\hline 3 & $01 X X$ & $01 X X, 05 X X$ & $01 X X, 06 X X$ & & & 1 & 1 & Lr-1.0/33.0 \\
\hline 4 & $01 X X$ & $01 X X, 05 X X$ & $01 X X, 10 x x$ & & & 10 & 10 & Lr-1.0/39.0 \\
\hline 5 & $01 X x$ & $01 X X, 07 X X$ & $01 X x$ & & & 1 & 1 & Lr-1.0/31.0 \\
\hline 6 & $01 X X, 04 X X$ & $01 X X, 04 X X$ & $01 X X, 04 X X$ & 6 & 1 & 8 & 15 & Lr-1.0/4.0 \\
\hline 7 & $01 X X, 07 X X$ & $01 X x$ & $01 X X$ & & & 1 & 1 & Lr-1.0/30.0 \\
\hline 8 & $01 X X, 08 X X$ & $01 X X, 05 X X$ & $01 X X, 10 X X$ & 2 & & & 2 & Lr-1.0/26.0 \\
\hline 9 & $01 X X, 08 X X$ & $01 X X, 06 X X$ & $01 X X, 05 X X$ & & & 1 & 1 & Lr-1.0/6.0 \\
\hline 10 & $01 X X, 08 X X$ & $01 X X, 06 X X$ & $01 X X, 12 X X$ & 3 & & 12 & 15 & Lr-1.0/22.0 \\
\hline 11 & $01 X X, 08 X X$ & $01 X X, 07 X X$ & $01 X X, 05 X X$ & & 2 & & 2 & Lr-1.0/7.0 \\
\hline 12 & 01XX, w09XX & $01 X X, 07 X X$ & $01 X X, 05 X X$ & & & 2 & 2 & Lr-1.0/28.0 \\
\hline 13 & 01XX, w09XX & $07 X X$ & $01 X X, 05 X X$ & & 4 & & 4 & Lr-11.0/28.0 \\
\hline 14 & $01 X X, 11 X X$ & $01 X X, 03 X X$ & $01 X X, 07 X X$ & 7 & & 1 & 8 & Lr-1.0/25.0 \\
\hline 15 & $01 X X, 11 X X$ & $01 X X, 05 X X$ & $01 X X$ & & 1 & & 1 & Lr-1.0/59.0 \\
\hline 16 & 01XX, rh03 & $01 X X, 06 X X$ & $01 X X, 05 X X$ & & 2 & 1 & 3 & Lr-1.0/21.0 \\
\hline 17 & $01 X X, 06 X X, 08 X X$ & $01 \times x$ & $01 X x$ & 6 & 3 & & 9 & Lr-1.0/27.0 \\
\hline 18 & 01XX, w09XX, 11XX & $03 X X, 07 X X$ & $05 X X, 07 X X$ & & 2 & & 2 & Lr-11.0/25.0 \\
\hline 19 & $02 X X, 04 X X, 07 X X$ & $04 X X$ & $02 X X, 04 X X$ & & 2 & & 2 & Lr-2.0/4.0 \\
\hline 20 & $02 X X, 07 X X, 11 X X$ & $03 X X, 04 X X$ & $02 X X, 07 X X$ & & 1 & & 1 & Lr-2.0/25.0 \\
\hline 21 & $04 X X$ & $03 X X, 04 X X$ & $04 X X, 06 X X$ & & 6 & & 6 & Lr-4.0/61.0 \\
\hline 22 & $04 x x$ & $04 X X$ & $04 x x$ & 6 & & 2 & 8 & Lr-4.0/4.0 \\
\hline 23 & $04 X X$ & $04 X X, 05 X X$ & 04XX, w09XX & & & 2 & 2 & Lr-4.0/29.0 \\
\hline 24 & $04 X X$ & $04 X X, 05 X X$ & 04XX, w08XX & & & 1 & 1 & Lr-4.0/5.0 \\
\hline 25 & $04 X X$ & $04 X X, 05 X X$ & $04 X X, 10 X X$ & & & 5 & 5 & Lr-4.0/39.0 \\
\hline 26 & $04 X X$ & $04 X X, 07 X X$ & $01 X X, 04 X X$ & & 4 & & 4 & Lr-4.0/31.0 \\
\hline 27 & $04 X X$ & $05 x x$ & $w 08 X X, 10 X X$ & & & 1 & 1 & Lr-5.0/39.0 \\
\hline 28 & $04 X X, 07 X X$ & $01 X X, 04 X X$ & $01 X X, 04 X X$ & & & 3 & 3 & Lr-4.0/30.0 \\
\hline 29 & $04 X X, 08 X X$ & $04 X X, 05 X X$ & $04 X X, 10 X X$ & 1 & & & 1 & Lr-4.0/26.0 \\
\hline 30 & $04 X X, 08 X X$ & $04 X X, 06 X X$ & $04 X X, 05 X X$ & & & 9 & 9 & Lr-4.0/6.0 \\
\hline 31 & $04 X X, 08 X X$ & $04 X X, 06 X X$ & $04 X X, 12 X X$ & 1 & 3 & 6 & 10 & Lr-4.0/22.0 \\
\hline 32 & $04 X X, 08 X X$ & $04 X X, 07 X X$ & $04 X X, 05 X X$ & & 5 & & 5 & Lr-4.0/7.0 \\
\hline 33 & 04XX, w09XX & $04 X X, 07 X X$ & $01 X X, 04 X X, 05 X X$ & & 1 & 4 & 5 & Lr-4.0/28.0 \\
\hline 34 & 04XX, w09XX & $05 X X, 07 X X$ & $01 X X, 05 X X, w 08 X X$ & & & 1 & 1 & Lr-5.0/28.0 \\
\hline 35 & $04 X X, 11 X X$ & $03 X X, 04 X X$ & $04 X X, 07 X X$ & 6 & 2 & & 8 & Lr-4.0/25.0 \\
\hline 36 & $04 X X, 11 X X$ & $04 X X$ & $04 X X$ & 5 & & & 5 & Lr-4.0/43.0 \\
\hline 37 & $04 X X, 11 X X$ & $04 X X, 05 X X$ & $04 X x$ & & 5 & & 5 & Lr-4.0/59.0 \\
\hline 38 & 04XX, rh03 & $04 X X, 06 X X$ & $04 X X, 05 X X$ & & 2 & 1 & 3 & Lr-4.0/21.0 \\
\hline 39 & $06 X X, 08 X X$ & $01 X X, 05 X X$ & $01 X X, 10 X X$ & & & 1 & 1 & Lr-27.0/39.0 \\
\hline 40 & $06 X X, 08 X X$ & $01 X X, 06 X X$ & $01 X X, 12 X x$ & & 1 & & 1 & Lr-22.0/27.0 \\
\hline 41 & 06XX, 08XX, 11XX & $01 X X, 03 X X$ & 01XX, 07XX & 1 & & & 1 & Lr-25.0/27.0 \\
\hline 42 & 07XX, w09XX & $04 X X, 07 X X$ & $01 X X, 02 X X, 05 X X$ & & & 1 & 1 & Lr-28.0/32.0 \\
\hline 43 & $08 X X$ & $04 X X, 06 X X$ & $05 X X, 12 X X$ & & & 1 & 1 & Lr-22.0/34.0 \\
\hline 44 & $08 X X$ & $05 X X, 06 X X$ & w09XX, 12XX & & & 3 & 3 & Lr-22.0/29.0 \\
\hline 45 & $08 \times x$ & $05 X X, 06 X X$ & $10 x x, 12 X x$ & & & 3 & 3 & Lr-22.0/39.0 \\
\hline 46 & $08 X X$ & $06 X X$ & $12 X X$ & & & 6 & 6 & Lr-22.0/22.0 \\
\hline 47 & $08 X X$ & $06 X X, 07 X X$ & $01 X X, 12 X X$ & & 2 & & 2 & Lr-22.0/31.0 \\
\hline 48 & 08XX, w09XX & $06 X X, 07 X X$ & $01 X X, 05 X X, 12 X X$ & & & 1 & 1 & Lr-22.0/28.0 \\
\hline 49 & $08 X X, 11 X X$ & $03 X X, 05 X X$ & $07 X X, 10 X X$ & 3 & & & 3 & Lr-25.0/26.0 \\
\hline 50 & $08 X X, 11 X X$ & $03 X X, 06 X X$ & $07 X X, 12 X X$ & 1 & & & 1 & Lr-22.0/25.0 \\
\hline 51 & 08XX, rh03 & $06 X X$ & $05 X X, 12 X X$ & & & 1 & 1 & Lr-21.0/22.0 \\
\hline 52 & w09XX & $05 X X, 07 X X$ & $01 X X, 05 X X, 10 X X$ & & & 2 & 2 & Lr-28.0/39.0 \\
\hline 53 & $11 X x$ & $03 X x$ & $07 X X$ & & 1 & & 1 & Lr-25.0/25.0 \\
\hline
\end{tabular}


Table 2 Continued.

\begin{tabular}{|c|c|c|c|c|c|c|c|c|}
\hline \multirow{2}{*}{$\begin{array}{l}\text { SLA class I } \\
\text { genotype }\end{array}$} & \multicolumn{3}{|c|}{ Allele specificity ${ }^{1}$} & \multicolumn{4}{|c|}{ No. of pigs } & \multirow{2}{*}{$\begin{array}{l}\text { Corresponding SLA } \\
\text { class I haplotype }\end{array}$} \\
\hline & $S L A-1$ & $S L A-3$ & $S L A-2$ & KSU & PCV & Big Pig & Total & \\
\hline 54 & $11 X X$, rh03 & $03 X X, 06 X X$ & $05 X X, 07 X X$ & & 1 & & 1 & Lr-21.0/25.0 \\
\hline 55 & Blank & $05 X X, 07 X X$ & 01XX, w09XX & & & 1 & 1 & Lr-29.0/31.0 \\
\hline \multirow[t]{2}{*}{56} & Blank & $05 X X, 07 X X$ & $01 X X, 10 X X$ & & & 1 & 1 & Lr-31.0/39.0 \\
\hline & & & Total number of pigs & 49 & 52 & 101 & 202 & \\
\hline
\end{tabular}

SLA, swine leucocyte antigen.

${ }^{1}$ Medium-/high-resolution specificity is not shown.

${ }^{2}$ Only confirmed in the KSU pigs by pedigree. Haplotypes were inferred in the PCV pigs and in the Big Pig as described in Materials and methods.

Table 3 Low-resolution SLA class I haplotypes identified in three experimental populations of outbred pigs.

\begin{tabular}{|c|c|c|c|c|c|c|c|}
\hline \multirow{2}{*}{$\begin{array}{l}\text { SLA class I } \\
\text { haplotype }\end{array}$} & \multicolumn{3}{|c|}{ SLA allele specificity ${ }^{1}$} & \multicolumn{4}{|c|}{ Haplotype frequency (\%) } \\
\hline & $S L A-1$ & $S L A-3$ & $S L A-2$ & KSU & PCV & Big Pig & Combined \\
\hline Lr-1.0 & $01 X x$ & $01 X X$ & $01 x x$ & 26.5 & 9.6 & 25.7 & 20.6 \\
\hline $\operatorname{Lr}-2.0$ & $02 X X, 07 X X$ & $04 X X^{2}$ & $02 x x$ & & 2.9 & & 1.0 \\
\hline Lr-4.0 & $04 X X$ & $04 X X$ & $04 X X$ & 31.6 & 29.8 & 21.3 & 27.6 \\
\hline Lr-5.0 & $04 X X$ & $05 x X$ & w08XX & & & 1.5 & 0.5 \\
\hline Lr-6.0 & $08 x x$ & $06 x x$ & $05 x x$ & & & 5.0 & 1.7 \\
\hline Lr-7.0 & $08 x x$ & $07 x x$ & $05 x x$ & & 6.7 & & 2.2 \\
\hline Lr-11.0 & 01XX, w09XX & $07 X X$ & $05 x x$ & & 5.8 & & 1.9 \\
\hline $\operatorname{Lr}-21.0$ & rh03 & $06 x x$ & $05 x x$ & & 4.8 & 1.5 & 2.1 \\
\hline Lr-22.0 & $08 X X$ & $06 x x$ & $12 X X$ & 5.1 & 5.8 & 19.3 & 10.1 \\
\hline Lr-25.0 & $11 x x$ & $03 x x$ & $07 X X$ & 18.4 & 7.7 & 0.5 & 8.9 \\
\hline Lr-26.0 & $08 \times x$ & $05 X X$ & $10 x x$ & 6.1 & & & 2.0 \\
\hline Lr-27.0 & $06 X X, 08 X X$ & $01 X X$ & $01 X X$ & 7.1 & 3.9 & 0.5 & 3.8 \\
\hline Lr-28.0 & w09xx & $07 X X$ & $01 X X, 05 X X$ & & 4.8 & 5.4 & 3.4 \\
\hline Lr-29.0 & Blank & $05 x x$ & w09xx & & & 3.0 & 1.0 \\
\hline Lr-30.0 & $07 X X$ & $01 X X$ & $01 X X$ & & & 2.0 & 0.7 \\
\hline Lr-31.0 & Blank & $07 X X$ & $01 X X$ & & 5.8 & 1.5 & 2.4 \\
\hline Lr-32.0 & $07 X X$ & $04 X X$ & $02 x X$ & & & 0.5 & 0.2 \\
\hline Lr-33.0 & $01 X X$ or Blank ${ }^{3}$ & $05 x x$ & $06 x x$ & & & 0.5 & 0.2 \\
\hline Lr-34.0 & Blank & $04 X X$ & $05 x x$ & & & 0.5 & 0.2 \\
\hline Lr-39.0 & Blank & $05 x X$ & $10 x x$ & & & 11.4 & 3.8 \\
\hline $\operatorname{Lr}-43.0$ & $11 x x$ & $04 X X$ & $04 x x$ & 5.1 & & & 1.7 \\
\hline Lr-59.0 & $11 x x$ & $05 x x$ & Blank $^{4}$ & & 5.8 & & 1.9 \\
\hline Lr-61.0 & Blank & $03 x x$ & $06 x x$ & & 6.7 & & 2.2 \\
\hline
\end{tabular}

SLA, swine leucocyte antigen.

${ }^{1}$ Medium-/high-resolution specificity is not shown.

${ }^{2}$ Probably due to the presence of $S L A-3{ }^{*} 04 X X$-like pseudogene as this haplotype did not appear to possess an expressed SLA-3 gene (Smith et al. 2005c; Sullivan et al. 1997).

${ }^{3}$ Ambiguity could not be resolved due to the detection of this haplotype in only one heterozygous animal.

${ }^{4}$ New SLA-2 allele (SLA-2*jh02) that was not covered by the typing primers used in this study.

in Hp-2.0 (Sullivan et al. 1997; Smith et al. 2005c), this suggests the possible presence of $S L A-3^{*} 04 X X-$ like pseudogene in this haplotype.

\section{Discussion}

In this study, we developed a simple, rapid and cost-effective method for typing classical SLA class I alleles in outbred pigs using the PCR-SSP strategy. This is one of the few reports on using molecular-based methods to survey SLA class I diversity in outbred commercial pigs. The PCR-SSP technique has been implemented extensively in human transplant-related tissue typing due to its high accuracy, efficiency and reproducibility (Schaffer \& Olerup 2001). It has been described for analysis of MHC polymorphism in a number of other mammalian species (Ellis et al. 1998; Wei 
et al. 2007). This methodology has also been described for SLA-typing several SLA-inbred pig herds (Martens et al. 2003; Ando et al. 2005; Ho et al. 2006) in which their SLA alleles and haplotypes were limited and first defined by SBT. The drawback of PCR-SSP is its limitation that it can type only alleles with known DNA sequences. In order to adapt this strategy to SLA-type outbred pigs with diverse and unknown immunogenetic backgrounds, we took advantage of the group assignments of SLA alleles based on sequence similarity and developed a set of PCR primers to distinguish alleles by the group-specific sequence motifs. This lowresolution typing approach eliminates the need to design a large number of allele-specific primers and allows the detection of almost all the class I alleles published to date in only half of a standard 96-well PCR plate.

A number of other molecular-based methods have been described for typing SLA genes. SBT of SLA alleles is the most direct and accurate method (Ando et al. 2003; Lee et al. 2005; Smith et al. 2005c; Ho et al. 2006). This approach usually requires cloning of the alleles to resolve heterozygous sequences, making it labour-intensive, timeconsuming and cost-prohibitive to be implemented in large scale SLA-typing studies. SBT is often more suitable for SLAtyping parental or founder breeding animals of pedigreed pig populations. The SLA types of the offspring can then be followed by other more effective methods, such as PCR-SSP, PCR-restriction fragment length polymorphism (PCR-RFLP) or microsatellite (MS) markers. PCR-RFLP analysis has been described to examine SLA allelic differences (Shia et al. 1995; Ando et al. 2005). This method of typing is generally fast, easy and relatively inexpensive to perform. However, the resolution greatly depends on the availability of restriction enzymes for differentiating specific polymorphic sites. As the number of polymorphisms assayed increases, the expected reaction patterns can also quickly become complicated and difficult to interpret. Haplotyping using MS markers within the MHC region has been described as a surrogate test for SLA loci (Nunez et al. 2004; Tanaka et al. 2005; Ando et al. 2008). The MS typing method is fast, easy, inexpensive to perform, and has been implemented widely for genetic mapping of QTL linked to production traits. However, the resolution of this method greatly depends on the availability and comprehensiveness of the markers in the region. The heterogeneity of the MS markers also does not necessarily correlate with SLA types. SLA typing by the MS method may be more appropriate for determining the compatibility between pedigreed animals, whereas typing of non-pedigreed outbred pigs using this method is more likely to give ambiguous results.

A total of 23 SLA class I haplotypes were identified in this study, although only the haplotypes in the KSU pigs could be confirmed by pedigree. The high-resolution equivalents of Lr-2.0, -11.0 and -59.0 detected in the PCV pigs were previously reported in multiple miniature pig breeds including the NIH, Sinclair, Hanford and the Korean native pigs (Smith et al. 2005b; Lee et al. 2008). This suggests that the PCV pigs may share some common genetic background with these breeds. The SLA class I haplotypes Lr-1.0, -4.0, $-22.0,-25.0$ and -27.0 were observed in all three experimental pig populations, while Lr-21.0, -28.0 and -31.0 were observed in two. Lr-1.0 and Lr-4.0 were identified as the most prevalent haplotypes in all three pig populations. Their high-resolution equivalents, Hp-1.0 and Hp-4.0, have been observed in multiple commercial and miniature pig breeds (Smith et al. 2005b; Ho et al. 2009). This high prevalence is also consistent with previous observations that their serologic equivalents, H01/H28 and H04, respectively, were very common in many commercial pig breeds such as the Large White, Landrace and Duroc (Renard et al. 1982, 1988). The serologic equivalent of Lr-21.0, H03, was also frequently observed in commercial pig breeds. Due to the lack of serologic association, the question of whether the other shared class I haplotypes are also common in the current commercial pig populations therefore requires further investigation.

Seven SLA class I specificities were not detected in this study. The SLA-2*jh02 in Lr-59.0 was not detected due to its recent addition to the SLA database and its unique sequence motifs that were not assayed by the current typing primers. The undetected SLA-1 specificities in Lr-29.0, -31.0, -34.0, -39.0, -61.0, and probably Lr-33.0, may also result from novel alleles with distinctive sequence motifs that are not covered. In addition, these undetected novel SLA-1 alleles may share similar sequence motifs with their respective SLA-2 and/or SLA-3 alleles on the same haplotype and therefore could not be resolved (i.e. cross-locus reactivity). Furthermore, these may result from primer insensitivity, as reference DNA samples were not available for evaluating a few primer pairs. An alternate explanation is that these SLA class I haplotypes do not possess expressed SLA-1 loci; growing evidence suggests that the number of expressed SLA loci is haplotype-dependent. This is demonstrated by the lack of expressed SLA-3, SLA-1 and SLA-6 antigens in haplotype Hp-2.0, -3.0 and -5.0, respectively (Sullivan et al. 1997; Smith et al. 2005c), accompanied with the suggested SLA-1 duplication in multiple class I haplotypes (Smith et al. 2005b; Ho et al. 2009). Further experiments to characterize these missing SLA-1 specificities at other polymorphic sites or sequencing of these loci are necessary for the improvement of the typing primers.

The KSU herd was originated by nine founding animals obtained commercially while the Big Pig samples were obtained from 17 litters of commercial pigs, making 18 and 68 , respectively, the maximum of SLA class I haplotypes that could potentially be detected. Yet only seven haplotypes (39\%) were observed in the KSU pigs and 16 (24\%) in the Big Pig samples. The limited SLA diversity observed in these two pig populations may simply result from the founder effect in which only a few SLA haplotypes were present in the founding animals. Alternatively, 
it may imply a limited SLA diversity in the current commercial pig breeding stock, perhaps resulting in part from selection for desirable production traits in modern agricultural practice. QTL linked to many favourable production traits are known to map close to the SLA complex (Pig QTL Database, http://www.animalgenome.org/QTLdb/ pig.html). Limited SLA class I diversity may have important implications in the susceptibility of commercial pigs to emerging pathogens and potential threats from agricultural terrorism. On the other hand, the SLA diversity observed in this study may not be an accurate or adequate estimate for the entire commercial pig population due to the relatively small sample size. Moreover, animals in these experimental pig populations were specifically bred (KSU and PCV) and chosen (Big Pig) for studies on swine responses to pathogens. Their SLA types, consequently, may have been unintentionally selected or inbred based on certain favourable genetic/phenotypic traits that are linked to the SLA complex. The SLA diversity observed in these experimental pigs may also have been influenced by the breeding schemes (e.g. number and relatedness of the founding boars and sows); this information was not known for most of the pigs evaluated in this study. Nevertheless, this study presents one of the first glimpses into the extent of SLA diversity in commercial pigs at the molecular level. It adds to our understanding of the allelic architecture of the SLA system and establishes a foundation for future studies in other pig populations.

In conclusion, we have developed a rapid and cost-effective SLA typing method using the PCR-SSP strategy for the detection of SLA class I alleles in outbred pig populations. This typing system demonstrated an accurate and unambiguous characterization of SLA class I alleles in outbred pigs. We suggest that it can facilitate the study of SLA influence in various swine immunological and pathophysiological conditions. This typing method may also provide insight on the development of effective vaccines and breeding of disease-resistant pigs in the context of SLA antigens for the improvement of swine health.

\section{Acknowledgements}

The authors wish to thank Drs John E. Butler (University of Iowa, Iowa City, IA), Kelly M. Lager (USDA Agriculture Research Service, Ames, IA), Carol R. Wyatt (Kansas State University, Manhattan, KS), and Ramon M. Molina and Jeffrey J. Zimmerman (Iowa State University, Ames, IA) for generously providing valuable DNA samples. The authors also thank Leah Pittmon and Lauren Winfree for expert technical assistance. This project was in part supported by USDA/NRI-CSREES grant 2004-35605-14197, 'Integrated Control and Elimination of Porcine Reproductive and Respiratory Syndrome Virus (PRRSV) in the U.S'. Y.-J. Lee was supported by the Korea Research Foundation (Grant No. KRF-2006-612-F00002).

\section{References}

Ando A., Kawata H., Shigenari A. et al. (2003) Genetic polymorphism of the swine major histocompatibility complex (SLA) class I genes, SLA-1, -2 and -3. Immunogenetics 55, 583-93.

Ando A., Ota M., Sada M. et al. (2005) Rapid assignment of the swine major histocompatibility complex (SLA) class I and II genotypes in Clawn miniature swine using PCR-SSP and PCRRFLP methods. Xenotransplantation 12, 121-6.

Ando A., Uenishi H., Kawata H., Tanaka-Matsuda M., Shigenari A., Flori L., Chardon P., Lunney J.K., Kulski J.K. \& Inoko H. (2008) Microsatellite diversity and crossover regions within homozygous and heterozygous SLA haplotypes of different pig breeds. Immunogenetics 60, 399-407.

Chardon P., Renard C., Rogel-Gaillard C. \& Vaiman M. (2000) The porcine major histocompatibility complex and related paralogous regions: a review. Genetics Selection Evolution 32, 109-28.

Demeure O., Sanchez M.P., Riquet J. et al. (2005) Exclusion of the swine leukocyte antigens as candidate region and reduction of the position interval for the Sus scrofa chromosome 7 QTL affecting growth and fatness. Journal of Animal Science 83, 1979-87.

Ellis S.A., Staines K.A., Stear M.J., Hensen E.J. \& Morrison W.I. (1998) DNA typing for BoLA class I using sequence-specific primers (PCR-SSP). European Journal of Immunogenetics 25, 365-70.

Ellis S.A., Bontrop R.E., Antczak D.F. et al. (2006) ISAG/IUIS-VIC Comparative MHC Nomenclature Committee report, 2005. Immunogenetics 57, 953-8.

Ho C.-S., Rochelle E.S., Martens G.W., Schook L.B. \& Smith D.M. (2006) Characterization of swine leukocyte antigen polymorphism by sequence-based and PCR-SSP methods in Meishan pigs. Immunogenetics 58, 873-82.

Ho C.-S., Lunney J.K., Ando A., Rogel-Gaillard C., Lee J.H., Schook L.B. \& Smith D.M. (2009) Nomenclature for factors of the SLA system, update 2008. Tissue Antigens (in press).

Lacey C., Wilkie B.N., Kennedy B.W. \& Mallard B.A. (1989) Genetic and other effects on bacterial phagocytosis and killing by cultured peripheral blood monocytes of SLA-defined miniature pigs. Animal Genetics 20, 371-81.

Lager K.M., Gauger P.C., Vincent A.L., Opriessnig T., Kehrli M.E. Jr \& Cheung A.K. (2007) Mortality in pigs given porcine circovirus type 2 subgroup 1 and 2 viruses derived from DNA clones. Veterinary Record 161, 428-9.

Lee J.H., Simond D., Hawthorne W.J., Walters S.N., Patel A.T., Smith D.M., O'Connell P.J. \& Moran C. (2005) Characterization of the swine major histocompatibility complex alleles at eight loci in Westran pigs. Xenotransplantation 12, 303-7.

Lee Y.J., Cho K.H., Kim M.J., Smith D.M., Ho C.S., Jung K.C., Jin D.I., Park C.S., Jeon J.T. \& Lee J.H. (2008) Sequence-based characterization of the eight SLA loci in Korean native pigs. International Journal of Immunogenetics 33, 362-74.

Lumsden J.S., Kennedy B.W., Mallard B.A. \& Wilkie B.N. (1993) The influence of the swine major histocompatibility genes on antibody and cell-mediated immune responses to immunization with an aromatic-dependent mutant of Salmonella typhimurium. Canadian Journal of Veterinary Research 57, 14-8.

Lunney J.K., Ho C.-S., Wysocki M. \& Smith D.M. (2008) Molecular genetics of the swine major histocompatibility complex, the SLA complex. Developmental and Comparative Immunology $35,333-4$. 
Madden K.B., Murrell K.D. \& Lunney J.K. (1990) Trichinella spiralis: major histocompatibility complex-associated elimination of encysted muscle larvae in swine. Experimental Parasitology 70, 443-51.

Mallard B.A., Wilkie B.N. \& Kennedy B.W. (1989a) Influence of major histocompatibility genes on serum hemolytic complement activity in miniature swine. American Journal of Veterinary Research 50, 359-63.

Mallard B.A., Wilkie B.N. \& Kennedy B.W. (1989b) The influence of the swine major histocompatibility genes (SLA) on variation in serum immunoglobulin (Ig) concentration. Veterinary Immunology and Immunopathology 21, 139-51.

Martens G.W., Lunney J.K., Baker J.E. \& Smith D.M. (2003) Rapid assignment of swine leukocyte antigen haplotypes in pedigreed herds using a polymerase chain reaction-based assay. Immunogenetics 55, 395-401.

Nunez Y., Ponz F. \& Gallego F.J. (2004) Microsatellite-based genotyping of the swine lymphocyte alloantigens (SLA) in miniature pigs. Research in Veterinary Science 77, 59-62.

Renard C., Chardon P. \& Vaiman M. (1982) The pig histocompatibility system SLA: serological study on a group of antigenic specificities. Animal Blood Groups and Biochemical Genetics 13, $161-77$.

Renard C., Kristensen B., Gautschi C., Hruban V., Fredholm M. \& Vaiman M. (1988) Joint report of the first international comparison test on swine lymphocyte alloantigens (SLA). Animal Genetics 19, 63-72.

Renard C., Hart E., Sehra H. et al. (2006) The genomic sequence and analysis of the swine major histocompatibility complex. Genomics 88, 96-110.

Robinson J., Waller M.J., Stoehr P. \& Marsh S.G. (2005) IPD - the Immuno Polymorphism Database. Nucleic Acids Research 33, D523-6.

Rothschild M.F., Chen H.L., Christian L.L., Lie W.R., Venier L., Cooper M., Briggs C. \& Warner C.M. (1984) Breed and swine lymphocyte antigen haplotype differences in agglutination titers following vaccination with B. bronchiseptica. Journal of Animal Science 59, 643-9.
Schaffer M. \& Olerup O. (2001) HLA-AB typing by polymerasechain reaction with sequence-specific primers: more accurate, less errors, and increased resolution compared to serological typing. Tissue Antigens 58, 299-307.

Shia Y.C., Bradshaw M., Rutherford M.S., Lewin H.A. \& Schook L.B. (1995) Polymerase chain reaction based genotyping for characterization of SLA-DQB and SLA-DRB alleles in domestic pigs. Animal Genetics 26, 91-100.

Smith D.M., Lunney J.K., Ho C.S., Martens G.W., Ando A., Lee J.H., Schook L., Renard C. \& Chardon P. (2005a) Nomenclature for factors of the swine leukocyte antigen class II system, 2005. Tissue Antigens 66, 623-39.

Smith D.M., Lunney J.K., Martens G.W., Ando A., Lee J.H., Ho C.S., Schook L., Renard C. \& Chardon P. (2005b) Nomenclature for factors of the SLA class-I system, 2004. Tissue Antigens 65, 13649.

Smith D.M., Martens G.W., Ho C.S. \& Asbury J.M. (2005c) DNA sequence based typing of swine leukocyte antigens in Yucatan miniature pigs. Xenotransplantation 12, 481-8.

Sullivan J.A., Oettinger H.F., Sachs D.H. \& Edge A.S. (1997) Analysis of polymorphism in porcine MHC class I genes: alterations in signals recognized by human cytotoxic lymphocytes. Journal of Immunology 159, 2318-26.

Tanaka M., Ando A., Renard C., Chardon P., Domukai M., Okumura N., Awata T. \& Uenishi H. (2005) Development of dense microsatellite markers in the entire SLA region and evaluation of their polymorphisms in porcine breeds. Immunogenetics $\mathbf{5 7 , 6 9 0 -}$ 6.

Tissot R.G., Beattie C.W. \& Amoss M.S. Jr (1989) The swine leucocyte antigen (SLA) complex and Sinclair swine cutaneous malignant melanoma. Animal Genetics 20, 51-7.

Vaiman M., Chardon P. \& Rothschild M.F. (1998) Porcine major histocompatibility complex. Revue Scientifique et Technique 17, 95-107.

Wei H., Wang H., Hou S., Hu S., Fan K., Fan X., Zhu T. \& Guo Y. (2007) DRB genotyping in cynomolgus monkeys from China using polymerase chain reaction-sequence-specific primers. Human Immunology 68, 135-44. 